\title{
Generalized cerebral atrophy seen on MRI in a naturally exposed animal model for creutzfeldt- jakob disease
}

\author{
Alexia L McKnight ${ }^{1 *}{ }^{*}$, Lawrence A Minkoff ${ }^{2 \dagger}$, Diane L Sutton ${ }^{3}$, Bruce V Thomsen ${ }^{4}$, Perry L Habecker ${ }^{5}$, \\ Raymond W Sweeney ${ }^{6}$, Gary Smith7, Constantin A Dasanu ${ }^{8}$, Thomas E Ichim ${ }^{9}$, Doru T Alexandrescu ${ }^{10}$, \\ Joel M Stutman ${ }^{11+}$
}

\begin{abstract}
Background: Magnetic resonance imaging has been used in the diagnosis of human prion diseases such as SCJD and VCJD, but patients are scanned only when clinical signs appear, often at the late stage of disease. This study attempts to answer the questions "Could MRI detect prion diseases before clinical symptoms appear?, and if so, with what confidence?"

Methods: Scrapie, the prion disease of sheep, was chosen for the study because sheep can fit into a human sized MRI scanner (and there were no large animal MRI scanners at the time of this study), and because the USDA had, at the time of the study, a sizeable sample of scrapie exposed sheep, which we were able to use for this purpose. 111 genetically susceptible sheep that were naturally exposed to scrapie were used in this study.

Results: Our MRI findings revealed no clear, consistent hyperintense or hypointense signal changes in the brain on either clinically affected or asymptomatic positive animals on any sequence. However, in all $37 \mathrm{PrP}{ }^{\text {Sc }}$ positive sheep (28 asymptomatic and 9 symptomatic), there was a greater ventricle to cerebrum area ratio on MRI compared to $74 \mathrm{PrP}^{\mathrm{SC}}$ negative sheep from the scrapie exposed flock and 6 control sheep from certified scrapie free flocks as defined by immunohistochemistry (IHC).

Conclusions: Our findings indicate that MRI imaging can detect diffuse cerebral atrophy in asymptomatic and symptomatic sheep infected with scrapie. Nine of these 37 positive sheep, including 2 one-year old animals, were $\mathrm{PrP}^{\mathrm{SC}}$ positive only in lymph tissues but $\mathrm{PrP}^{\mathrm{SC}}$ negative in the brain. This suggests either 1 ) that the cerebral atrophy/neuronal loss is not directly related to the accumulation of $\mathrm{PrP}^{\mathrm{Sc}}$ within the brain or 2) that the amount of $\mathrm{PrP}^{\mathrm{Sc}}$ in the brain is below the detectable limits of the utilized immunohistochemistry assay. The significance of these findings remains to be confirmed in human subjects with CJD.
\end{abstract}

\section{Background}

Scrapie was first reported in 1730 in sheep and goats and is the longest known transmissible spongiform encephalopathy (TSE) [1]. In the past two decades, TSEs have received much attention since ingestion of bovine spongiform encephalopathy (BSE) infected beef was causally linked to the variant form of CJD (vCJD) [2]. These TSE diseases are progressively debilitating and invariably

\footnotetext{
* Correspondence: alexia@mcknightinsight.com

† Contributed equally

'Assistant Professor of Radiology, University of Pennsylvania School of Veterinary Medicine, New Bolton Center, Kennett Square, PA 19348, USA Full list of author information is available at the end of the article
}

fatal neurodegenerative diseases that have very long incubation periods and unique neuropathological changes. The most widely accepted cause of the TSE diseases is an abnormal prion protein, identified as $\mathrm{PrP}^{\mathrm{Sc}}$ in the case of scrapie, which is a stereoisomer of the normal prion protein $\left(\operatorname{PrP}^{\mathrm{C}}\right)$.

Ante-mortem diagnosis of the TSE diseases, in general, has proven to be quite challenging. MRI has been useful in CJD patients - with both the sporadic and variant forms. It is helpful in the exclusion of other neurodegenerative diseases as well as, in some cases, the positive diagnosis of sCJD or vCJD [3-6]. For example,

\section{C) Biomed Central}


in a study of 162 sCJD cases, bilateral basal ganglia hyperintensity was found to be $67 \%$ sensitive and $94 \%$ specific. MRI findings included hyperintense alterations and/or brain atrophy, alone or in combination with each other. A normal MRI without any hyperintense changes or atrophy was seen in $27.2 \%(44 / 162)$ patients [3]. A bilaterally symmetric hyperintense pulvinar, or the "hockey stick sign", is reported to be present in $80 \%$ of vCJD patients in some studies [4,5,7]. CJD patients, however, present with clinical symptoms at a relatively late stage of the disease.

MRI abnormalities are reported in pre-symptomatic mice experimentally infected (intraperitoneally) with scrapie. The study was performed at $9.4 \mathrm{~T}$, and a hyperintense septum and hippocampus were seen at 120 days post infection, approximately 60 days prior to the onset of clinical signs. Additional cortical and thalamic abnormalities were seen at 180 days post infection, when clinical signs became apparent [8].

Other MRI studies by Chung et al. in rodent scrapie models correlate MRI signal changes to neuoropathology $[9,10]$. One hamster model, performed at $4.7 \mathrm{~T}$, with scrapie induced intra-cerebral injections revealed a correlation with increased $\mathrm{T} 2$ signal and gliosis, and decreased $\mathrm{T} 2$ signal with vacuolization. In some areas with marked gliosis and vacuolization, no MRI signal changes were seen suggesting a T2 cancelling effect [10].

In contrast to Chung's findings in hamsters, Haik et al. found no association of MRI signal change in two CJD patients with gliosis and no clear association with spongiform change. There was, however, strong correlation of MRI signal change with accumulation of $\mathrm{PrP}^{\mathrm{Sc}}$ in the both the sCJD and the vCJD patient [11].

Unlike experimentally induced scrapie rodent models that have a different course of disease than natural infection and CJD patients that present with an advanced stage of disease, a naturally exposed scrapie flock is typically composed of sheep in various stages of disease. For this reason, these animals are considered a good model to study MRI findings in scrapie as a model for the TSE diseases. Our objective was to study the consistent MRI findings in a large flock of scrapie positive animals as confirmed by immunohistochemistry. The purpose was twofold: 1) to better understand TSE diseases by evaluating the MRI finding in naturally infected sheep, and 2) to assess the accuracy of MRI in the detection of TSE in both symptomatic and asymptomatic sheep.

\section{Methods}

\section{Flock information}

One hundred eleven scrapie-exposed sheep with the scrapie susceptible $\mathrm{QQ}_{171}$ genotype were used in this study. The sheep originated from a single commercial scrapie flock in the Midwest United States that was to be depopulated for regulatory reasons. The flock was comprised of 62 black faced breeds (24 Hampshire, 39 Suffolk, 1 unknown black faced), 37 Western white faced sheep which entered the flock as adults prior to this study, and 12 brockel faced sheep born of the white faced ewes and black faced rams. The sheep ranged in age from 1-9 years old with the oldest sheep primarily the Western white faced breed.

Six additional sheep were purchased from two separate certified scrapie free flocks; five were black faced and one was white faced. They ranged in age from 2-8 years and served as known negative controls.

\section{MRI Examinations - Part I}

Brain MR examinations were initially done on the 6 negative control animals and the 24 scrapie positive sheep which were identified by an immunohistochemistry test of surgically collected $3^{\text {rd }}$ eyelid lymph tissue from the 76 black and brockel faced sheep [12,13]. Each animal was scanned live under general anaesthesia and then recovered with the exception of the three most clinically affected animals, which were euthanized after the MRI exam, and one sheep that died during induction. We used a mobile 1T GE Signa LX MRI system (General Electric, Milwaukee, WI) with the general purpose flex coil wrapped around the dorsal and lateral aspects of the head. The following pulse sequences were obtained: T1- and T2-weighted fast spin echo, proton density (PD), inversion recovery (IR), fluid attenuated inversion recovery (FLAIR), and diffusion weighted imaging (DWI). The slice parameters were $3 \mathrm{~mm}$ thickness, 0 gap, $14 \mathrm{~cm}$ FOV for PD, T1, T2, IR, and FLAIR; for DWI $4 \mathrm{~mm}$ thickness, 0 gap, $22 \mathrm{~cm}$ FOV. Where possible 23 slices in the each of the axial, sagittal, and coronal planes were obtained.

\section{MRI Examinations - Part II}

Based on the findings from Part I, all 113 remaining sheep were examined or re-examined by the same MRI protocol (with the exception of the T1 and FLAIR sequences) immediately following euthanasia.

\section{Quantitative Analysis}

Lateral ventricle to cerebrum area ratios (V/C ratio) were calculated in all sheep. The $\mathrm{V} / \mathrm{C}$ ratio is calculated by the following formula:

\footnotetext{
Lateral ventricle to cerebrum Ratio $=$ Lateral ventricle area / (cerebrum area lateral ventricle area) $* 100$ or,

more concisely: $\mathrm{V} / \mathrm{C}=\mathrm{A}_{\mathrm{V}} /\left(\mathrm{A}_{\mathrm{C}}-\mathrm{A}_{\mathrm{V}}\right) * 100$ and is reported as a percent.
}

It is the area of the lateral ventricle normalized to the area of the sheep's cerebrum area as imaged in that sagittal slice, and the value is reported as a per cent. It 
is an effort to measure the size of this sheep's lateral ventricle area as related to its own cerebrum area (without the ventricle area included in the cerebrum area). On the FSE T2 weighted sequences, a sagittal slice 3-5 $\mathrm{mm}$ off midline that had the largest lateral ventricular area was used for the measurements. As shown in Figure 1 , regions of interest were drawn around the border of the lateral ventricle and, in the same slice, around the cerebrum.

Two scientists measured the areas using two different methods on different computers with no communication between them regarding their results. The scientists did their work in separate locations at different times with no communication regarding the $\mathrm{V} / \mathrm{C}$ results. Both used mouse pointers to trace the outlines of the lateral ventricle and the cerebrum in the same slice as defined above.

Scientist A used Adobe's Photoshop software and their "Magnetic Lasso" technology with the following parameters: feather = zero pixels; anti-aliased $=$ on; width = 3 pixels; edge contrast $=100 \%$, and frequency $=100$. Scientist B wrote his own software code in Microsoft Visual Basic 6.0 with DicomObjects.ocx as the DICOM interface and created a routine which counts pixels inside bounded planar regions. Dicomobjects.ocx is a library of compiled software enabling DICOM files to be studied using many different higher level programming languages for control. http://www.medicalconnections. co.uk.

Both techniques used exactly the same definition of the boundary between the cerebrum from the cerebellum, the only place in the slices of interest in which the boundary was less clear than all other tissue boundaries:

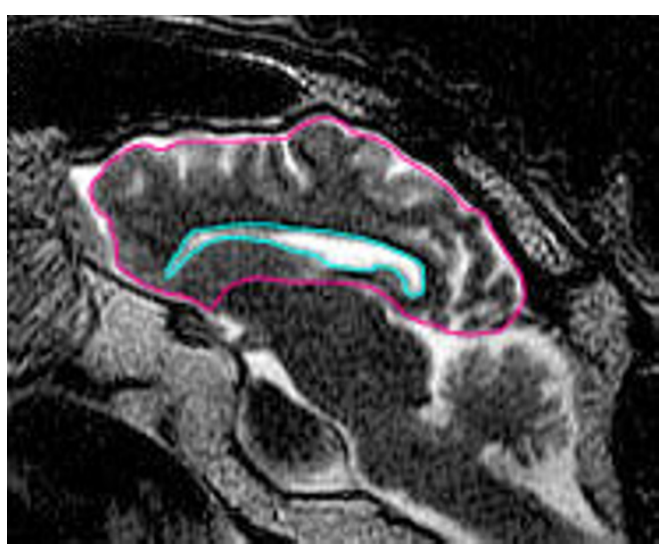

Figure 1 The quantitative analysis in this study was performed by outlining the perimeter of the lateral ventricle and the cerebrum on a sagittal slice 3-5 $\mathrm{mm}$ from midline where the largest area of lateral ventricle was present. The areas were determined and the ventricle to cerebrum ratio (V/C ratio) was then calculated. this required a line drawing rule over that narrow region, which rule was used by both. Resulting percentages were similar, only varying by a small multiplicative constant; and, finally, both obtained similar graphs.

For the total data set of 117 sheep, the inter-observer reliability (correlation coefficient) between the scientist A and scientist B was 0.85 by the Pearson Product Moment Method and 0.87 by the Spearman Rank Order Method.

\section{Laboratory analysis}

Scrapie testing by immunohistochemistry procedures followed the standard protocols used in the United States Department of Agriculture (USDA) scrapie eradication program and are similar to those described previously [13]. The pre-mortem third eyelid tissues were evaluated at the University of Wyoming (EW) and postmortem sections of medulla at the obex, medial retropharyngeal lymph node and tonsil were examined at the National Veterinary Services Laboratory in Ames, IA (BT). Briefly, tissue sections were deparaffinized, rehydrated, treated with 95\% formic acid (lymph tissue only) and then autoclaved in an antigen retrieval solution obtained from DakoCytomation, Carpinteria, CA, USA [14]. The sections were stained with an automated immunohistochemistry system (by Ventana Medical Systems, Tucson, AZ USA) which used a mixture of two monoclonal antibodies, F89/160.1.5 and F99/97.6.1, to detect prion protein [13]. Known positive and negative tissue samples were run as controls for each group of slides. The slides were interpreted independently of the MRI results. Later, additional areas of the brain (4 areas of the cerebrum and 1 section each of the thalamus, rostral colliculus, pons and cerebellum) were examined by IHC on eight of the nine animals which were found to be positive only on lymph tissue and negative on brain samples at the level of the obex.

All sheep were genotyped at codons 171, 136, and 154 . There were only 4 genotypes present in the 117 sheep: 97 AARRQQ; 4 AARHQQ; 4 AARRQR; and 12 AVRRQQ.

\section{Clinical examinations}

All 111 scrapie exposed sheep were evaluated clinically for neurological signs consistent with scrapie. The 24 eyelid positive animals were more thoroughly examined by recording the following parameters: body condition score, percent wool loss, presence of ataxia, and trembling.

\section{Statistical analysis}

The extent to which the MRI results discriminate between "scrapie" and "not scrapie" was evaluated using a receiver operating characteristic (ROC) curve 
consisting of a graph of sensitivity versus one minus specificity as the cutoff is varied. The parameters and characteristics of the ROC curve was estimated from the data using STATA (Statacorp, 2001). The area under the ROC curve is used as a summary measure of the extent of the discrimination [15].

\section{Approvals}

All aspects of this study were approved by the University of Pennsylvania's Institutional Animal Care and Use Committee, Environmental Health and Radiation Safety, and the Pennsylvania Department of Agriculture.

\section{Results}

The clinical signs of scrapie, trembling and ataxia with various combinations of wool loss and/or thin body condition score, were only identified in 9 sheep. The other 102 sheep in the infected flock showed no detectable signs consistent with scrapie. IHC testing found 37 out of 111 sheep positive for scrapie with 9 of 37 sheep positive only on lymph tissues. Eight of these 9 lymphonly positive sheep remained $\operatorname{PrP}^{\mathrm{Sc}}$ negative following additional IHC testing on multiple areas of the brain. Additional brain samples were unavailable for testing on the single remaining animal.

The third eyelid test identified 24/76 (31\%) sheep as $\operatorname{PrP}^{\mathrm{Sc}}$ positive. Performing the eyelid test allowed an antemortem diagnosis to identify several scrapie infected sheep. In Part I of the study when brain MRI exams of these eyelid positive animals were compared to the 6 control animals, no clear, consistent MRI signal changes were noted in the brain of either the 9 clinically affected or the 15 asymptomatic sheep on any pulse sequence. As seen in Figure 2, the most severely affected clinical animals had hyperintense adipose tissue, predominantly within the medullary cavity of the skull and around the retropharyngeal lymph nodes, corresponding with serous atrophy secondary to emaciation. There was also mild subjective enlargement of the lateral ventricles with sulcal prominence in the most clinically affected sheep indicative of diffuse cerebral atrophy (Figure 2). This finding prompted quantitative evaluation in all sheep.

The results of the quantitative analysis following Part II of the study are shown in Figures 3 and 4. The 37 $\mathrm{PrP}^{\mathrm{Sc}}$ positive sheep had larger $\mathrm{V} / \mathrm{C}$ ratios compared to the $\operatorname{PrP}^{\mathrm{Sc}}$ negative sheep (Figure 3). Interestingly, 9 of these 37 sheep, including 2 one-year olds, were $\operatorname{PrP}^{\mathrm{Sc}}$ positive in the retropharyngeal lymph nodes and/or tonsils but negative in the brain (Figure 4). As seen in Figure 4 , no correlation with the $\mathrm{V} / \mathrm{C}$ ratios with age was seen. Almost all animals with a V/C ratio over 15\% showed clinical symptoms of scrapie.

The $37 \mathrm{PrP}^{\mathrm{Sc}}$ positive sheep fall into the following genotypes: 36 of 97 AARRQQ; 0 of 4 AARHQQ; 0 of 4
AARRQR; and 1 of 12 AVRRQQ. Every score above $10.4 \%$ corresponded to a $\operatorname{PrP}^{\mathrm{Sc}}$ positive and every score below $9.5 \%$ corresponded to a negative: there was only a $10.25 \%$ overlap in scores, and most importantly only $8.75 \%$ false negatives when all scores are considered and the above cutoffs are not used.

In addition, it is noteworthy that the AVRRQQ subjects are, despite their relatively small $\mathrm{N}$, the most ambiguous, in that 5 of the 11 negatives with this genotype fall into the upper quartile of all negative scores; this 'leaning' towards the high end of the negative distribution, might serve to suggest that over time this genotype might turn out to be the most likely to shift from negative to positive, and in future work should receive special attention regarding possible false negatives.

The ROC curve is shown in Figure 5. The area under the curve was 0.99 (95\% confidence interval, 0.98-1.00). As described in Hosmer and Lemeshow (page 162), this is in the "outstanding discrimination" range [15].

\section{Discussion}

The 111 scrapie exposed QQ sheep used in this study are from a single commercial flock in the Midwest United States that had a high prevalence of infection $(33 \%$ of the QQ animals were $\operatorname{PrP}^{\mathrm{Sc}}$ positive on post-mortem $\mathrm{IHC}$ ). Because there was a wide range in ages, multiple breeds, and clinical stages of the disease progression, this flock was considered a good model for evaluating MRI findings in scrapie positive sheep. The MRI findings correlated with IHC results in each of the breeds examined. The scrapie associated MRI changes detected subclinically infected animals and also detected 10 animals which were not identified by the current antemortem third eyelid test.

MRI signal abnormalities were not seen consistently on T2, FLAIR, or PD weighted images in this flock as reported in CJD patients and rodent scrapie models. Although similar inconsistencies are also seen in people, hyperintense changes in this study were a rare finding. Reasons for the hyperintense change in some animals and not others remain unclear. Meissner et al. found a correlation between the presence and absence of MRI findings and the CJD genotype in human patients [6]. The genotypes of this flock are very homogeneous and could explain the relative uniform lack of MRI signal abnormality.

The animals with the most severe clinical signs had the highest ventricular to cerebrum ratios; almost all animals with a $\mathrm{V} / \mathrm{C}$ ratio over $15 \%$ showed clinical symptoms of scrapie. For this reason, we believe that enlarged ventricular to cerebral ratios may be positively correlated with disease progression. Similar correlations between brain atrophy as seen on MRI and progression of clinical disease have also been reported in other 


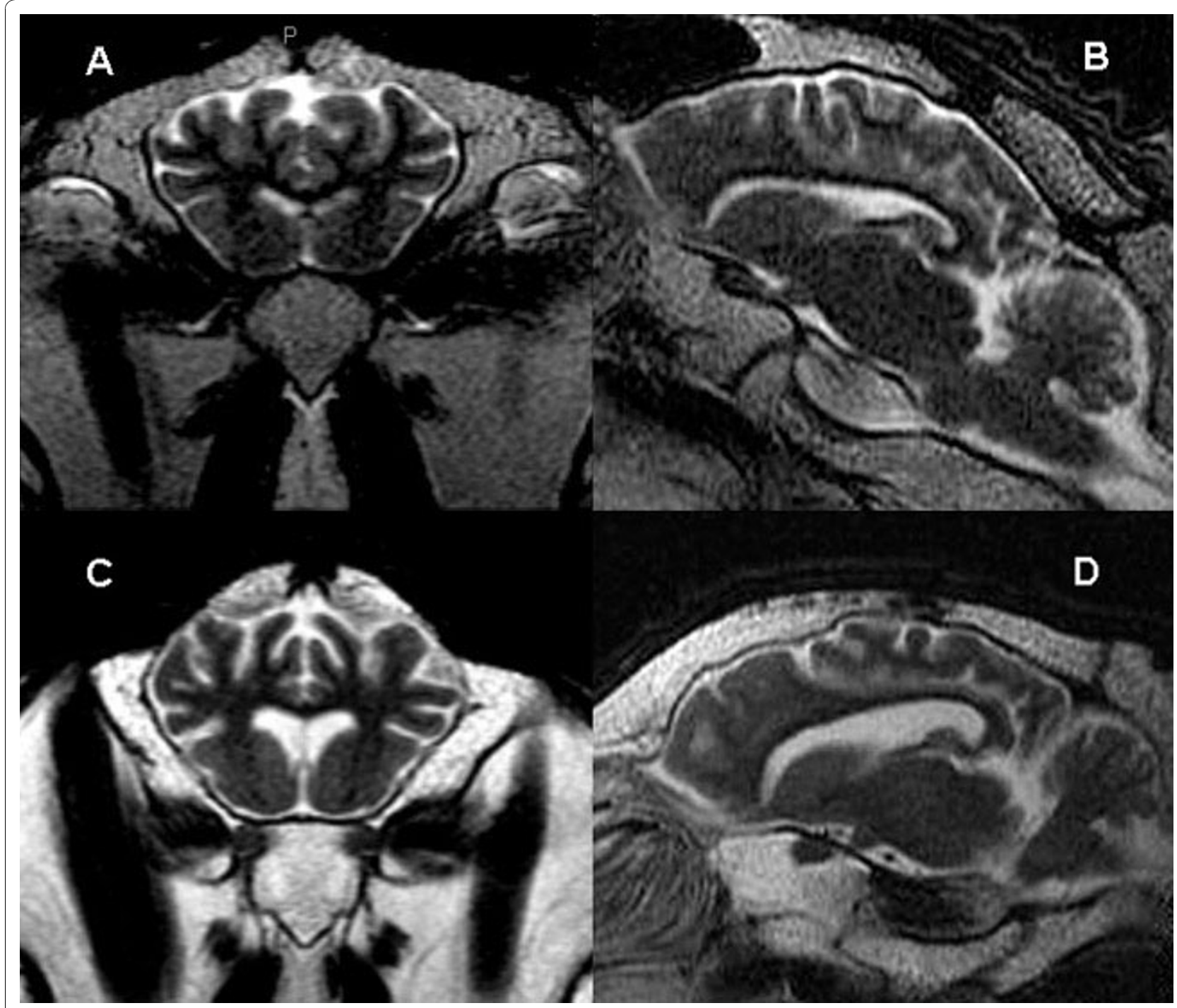

Figure 2 Axial and sagittal MR images of a normal control sheep ( $A$ and B) compared to the most clinically affected animal in the study (C and D). The sulcal prominence and enlarged lateral ventricles indicative of diffuse cerebral atrophy are seen in the scrapie affected sheep.

neurodegenerative diseases such as Alzheimer's disease [16] and multiple sclerosis $[17,18]$.

Ventricular enlargement with sulcal prominence is typical of brain atrophy on MRI examinations $[16,19,20]$. This observation in the most clinically affected animals suggested similar evidence of cerebral atrophy/neuronal loss that has been reported in CJD patients[3] and a rodent scrapie model[10] with advanced disease. Particularly noteworthy was the finding, following quantitative analysis of all 117 sheep (111 scrapie exposed and 6 normal controls) that cerebral atrophy was a consistent finding in the $37 \mathrm{PrP}^{\mathrm{Sc}}$ animals, even among the asymptomatic sheep and, of particular interest, in the 2 positive one-year old sheep. The quantification of certain brain parameters on MR images, such as the $\mathrm{V} / \mathrm{C}$ ratio as used in this study, may be considered as an ante-mortem tool for live animals at risk for scrapie, including young animals.

The pathophysiologic process that would explain diffuse cerebral atrophy in young asymptomatic sheep is unclear. The progression of scrapie in the naturally infected animal begins with an oral infection. Particularly susceptible in the perinatal period, lambs first show evidence of $\operatorname{PrP}^{\mathrm{Sc}}$ in the Peyer's patches, medial retropharyngeal lymph nodes, mesenteric lymph nodes, and tonsils about 2-5 months after birth [21-23] In approximately 12-18 months, but as early as 9-10 months, $\mathrm{PrP}^{\mathrm{Sc}}$ enters the central nervous system and can be first found in the obex of the medulla and the T8-T10 thoracic spinal cord segments [22,24] At the terminal stage 

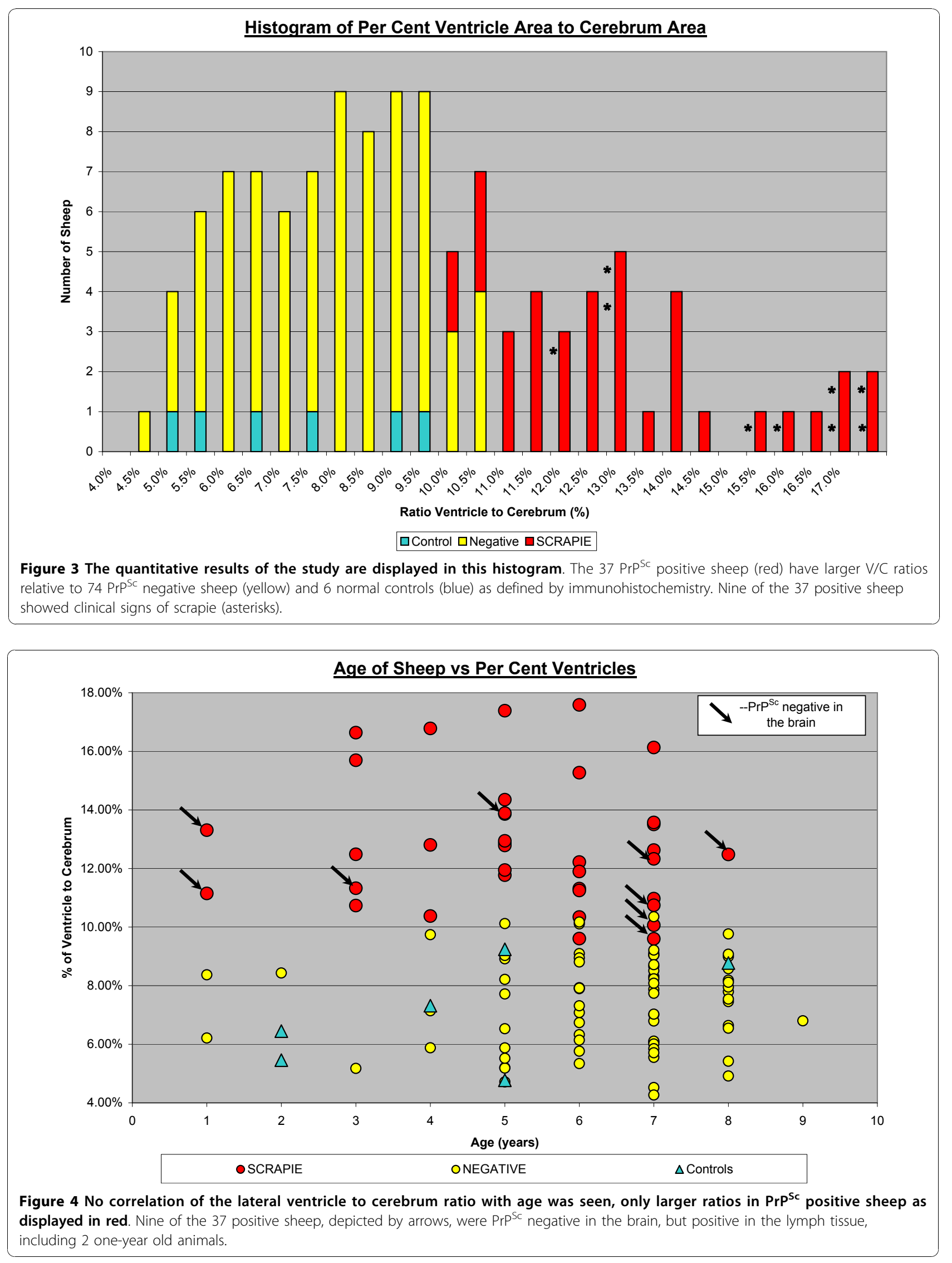


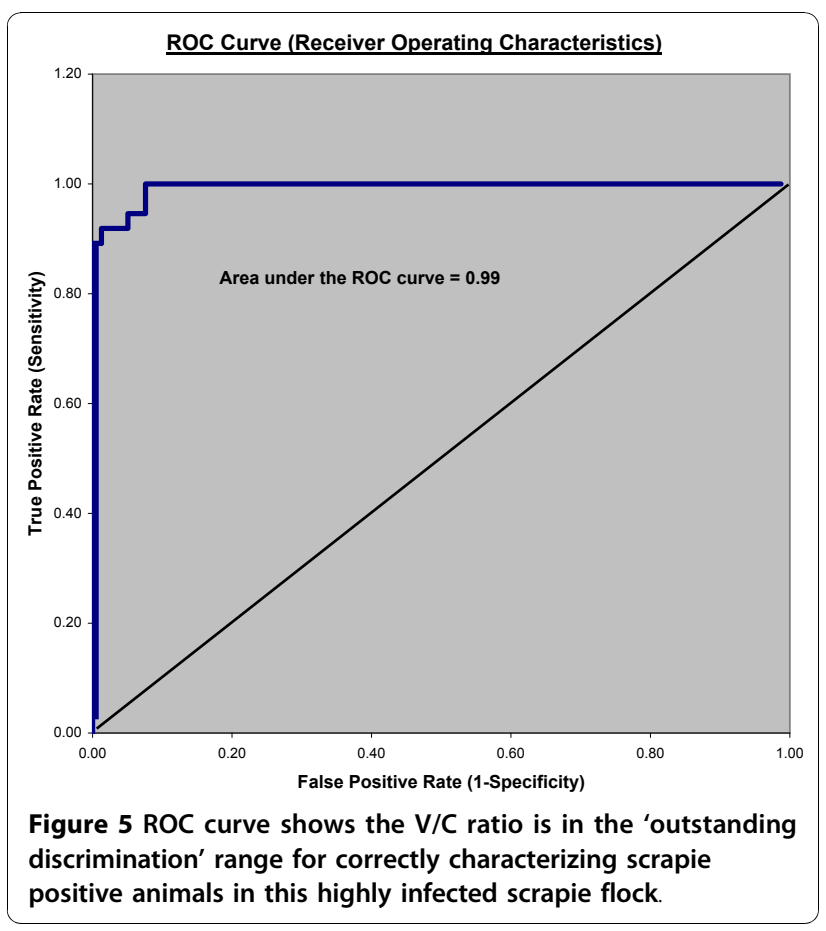

of disease in clinically affected animals, usually between 2-5 years of age, neuropil vacuolation, astrocytosis, neuronal loss, and shrunken 'dark neurons' are seen in areas rostral to the medulla [25]. These histopathological findings are reported to be within the neocortex of the cerebrum (particularly centered around the superior frontal lobe gyrus) as well as the diencephalon and mesencephalon, cerebellum, and brain stem [25].

However, as shown in the pathogenesis studies, $\mathrm{PrP}^{\mathrm{Sc}}$ within the cerebrum in asymptomatic sheep is not an expected finding $[21,22,24,25]$. Routine screening for scrapie on $\mathrm{IHC}$ is performed at the level of the obex of the medulla, because identification of $\operatorname{PrP}^{\mathrm{Sc}}$ at this site has shown to be most sensitive for detection of scrapie in the earliest stages of CNS involvement [26,27]. Ersdal et al. found evidence of $\operatorname{PrP}^{\mathrm{Sc}}$ in the cerebellum of 1/17 sheep and not in the obex [24]. Accumulation of $\operatorname{PrP}^{\mathrm{Sc}}$, spongiform change and/or astrogliosis rostral to the obex in asymptomatic sheep was not reported and considered to be unusual [24,26-30]. Therefore, diffuse cerebral atrophy in the 28 asymptomatic sheep in this study is difficult to explain.

Furthermore, 9 of the $37 \mathrm{PrP}^{\mathrm{Sc}}$ positive sheep in this study that had higher $\mathrm{V} / \mathrm{C}$ ratios compared to $\operatorname{PrP} \mathrm{P}^{\mathrm{Sc}}$ negative sheep were only $\mathrm{PrP}^{\mathrm{Sc}}$ positive in lymphoid tissue, not in the obex. Additional immunohistochemistry was negative on areas of the brain rostral to the obex in the 8 re-tested animals, which is consistent with the literature that the obex negative $\operatorname{PrP}^{\mathrm{Sc}}$ sheep are in an early stage of disease that has not yet reached the central nervous system [22]. Two possible explanations are that the diffuse cerebral atrophy in this scrapie flock is either not directly related to the accumulation of $\operatorname{Pr} \mathrm{P}^{\mathrm{Sc}}$ in the brain or that low levels of $\operatorname{PrP}^{\mathrm{Sc}}$ in the obex are present but simply undetected by the IHC testing.

\section{Conclusions}

We found a greater lateral ventricle to cerebrum area ratio in $\mathrm{PrP}^{\mathrm{Sc}}$ positive sheep compared to $\operatorname{PrP}^{\mathrm{Sc}}$ negative sheep in this large, naturally scrapie infected flock. There was no age correlation with the $\mathrm{V} / \mathrm{C}$ ratios, only higher ratios in more clinically affected sheep in advanced disease. The results of this study indicate that there is diffuse cerebral atrophy/neuronal loss seen with MRI in naturally infected scrapie sheep in young animals and prior to the onset of clinical signs that appears related to the progression of the disease. These results also suggest that the cerebral atrophy/neuronal loss is not directly related to the accumulation of $\operatorname{PrP}^{\mathrm{Sc}}$ within the brain, or that the amount of $\operatorname{PrP}^{\mathrm{Sc}}$ in the brain is below the detectable limits of the immunohistochemistry assay. The significance of these findings remains to be confirmed in human subjects with CJD.

\section{Acknowledgements}

The authors would like to acknowledge Dr. Elizabeth Williams at the Wyoming State Veterinary Laboratory for evaluating the third eyelid tissues, and Patricia Meinhardt and Norma Newton from National Veterinary Services Laboratories in Ames, lowa for the genotypic evaluations.

The authors heartily thank the faculty and staff of New Bolton Center's Anesthesia and Pathology, as well as the MRI technologists and farm assistants for all their help with this study. Nigel Watson of the New Bolton Pathology Department who did consistently precise dissection work on the obex and nodes of all 117 sheep deserves special recognition.

Acknowledgement is gratefully tendered to Dr. David Harvey, Genera Manager of Medical Connections, UK for very intelligent software assists with Dicomobjects.ocx. The authors are very grateful for the insightful statistical assistance, relative to the genotype analysis as well as the inter-observer reliability, provided by Dr. Leonard A. Rosenblum (Professor of Experimental Psychology (Retired), Downstate Medical Center, Brooklyn, NY).

Thank you also to the Louis and Lena Minkoff Foundation for the generous gift that funded this project. Dr. Lawrence Minkoff is the president of the Foundation and an author of this paper.

\section{Author details}

'Assistant Professor of Radiology, University of Pennsylvania School of Veterinary Medicine, New Bolton Center, Kennett Square, PA 19348, USA. ${ }^{2}$ Executive Vice President, Fonar Corporation, Marcus Drive, Melville, NY, USA. ${ }^{3}$ National Scrapie Program Coordinator, United States Department of Agriculture, Animal and Plant Health Inspection Service, Veterinary Services, Riverdale, MD 20737, USA. ${ }^{4}$ United States Department of Agriculture, Animal and Plant Health Inspection Service, Veterinary Services Laboratories, Ames, IA 50010, USA. ${ }^{5}$ Professor, The Pennsylvania Animal Diagnostic Laboratory System at New Bolton Center and the Laboratory of Pathology and Toxicology, School of Veterinary Medicine, University of Pennsylvania, 382 W. Street Road, Kennett Square, Pennsylvania 19348, USA. ${ }^{6}$ Professor of Medicine \& Chief, Section of Medicine, Department of Clinical Studies, New Bolton Center, 382 West Street Road, Kennett Square, PA 19348, USA. 7Professor of Population Biology and Epidemiology \& Chief, Section of Epidemiology and Public Health, Department of Clinical Studies, New Bolton Center, 382 West Street Road, Kennett Square, PA 19348, USA. ${ }^{8}$ Saint Francis Hospital and Medical Center, Hartford, CT 06105, USA. ${ }^{9}$ Medistem Inc., San Diego, CA 92101, USA. ${ }^{10}$ Georgetown Dermatology, Washington, DC 20010, USA. ${ }^{11}$ Professor and Chairman (Retired), Medical Computer Science 
Program, College of Health Related Professions, Downstate Medical Center, State University of New York, Brooklyn, NY, USA.

\section{Authors' contributions}

ALM-Primary Author, Study Staffing, Study Management. LAM-MRI data acquisition and analysis, JMS-MRI data acquisition and analysis, BVTImmunohistochemistry analysis at NVSL, PLH-Pathological assistance at New Bolton Center, RWS-Clinical assessment and medical care of flock, GSStatistical analysis, CAD-Clinical assessment and integration of the obtained data, TEI- Integration of the data, DTA-Clinical integration of the data, DLSAcquisition and coordination of infected flock. All authors have read and approved the final manuscript.

\section{Competing interests}

The Authors declare that they have no competing interests.

Received: 1 April 2010 Accepted: 26 November 2010 Published: 26 November 2010

\section{References}

1. Woolhouse ME, Coen P, Matthews L, Foster JD, Elsen JM, Lewis RM, Haydon DT, Hunter N: A centuries-long epidemic of scrapie in British sheep? Trends in Microbiology 2001, 9:67-70

2. Bruce ME, Will RG, Ironside JW, McConnell I, Drummond D, Suttie A McCardle L, Chree A, Hope J, Birkett C, et al: Transmissions to mice indicate that 'new variant' CJD is caused by the BSE agent. Nature 1997, 389:498-501

3. Schroter A, Zerr I, Henkel K, Tschampa HJ, Finkenstaedt M, Poser S: Magnetic resonance imaging in the clinical diagnosis of CreutzfeldtJakob disease. Arch Neurol 2000, 57:1751-1757.

4. Collie DA: The role of MRI in the diagnosis of sporadic and variant Creutzfeldt-Jakob disease. Jbr-Btr 2001, 84:143-146.

5. Collie DA, Summers DM, Sellar RJ, Ironside JW, Cooper S, Zeidler M, Knight R, Will RG: Diagnosing variant Creutzfeldt-Jakob disease with the pulvinar sign: MR imaging findings in 86 neuropathologically confirmed cases. Ajnr: American Journal of Neuroradiology 2003, 24:1560-1569.

6. Meissner B, Kortner K, Bartl M, Jastrow U, Mollenhauer B, Schroter A, Finkenstaedt M, Windl O, Poser S, Kretzschmar HA, Zerr I: Sporadic Creutzfeldt-Jakob disease: magnetic resonance imaging and clinical findings. Neurology 2004, 63:450-456.

7. Collie DA, Sellar RJ, Zeidler M, Colchester AC, Knight R, Will RG: MRI of Creutzfeldt-Jakob disease: imaging features and recommended MR protocol. Clinical Radiology 2001, 56:726-739.

8. Collie DA: The role of MRI in the diagnosis of sporadic and variant Creutzfeldt-Jakob disease. Jbr-Btr: Organe de la Societe Royale Belge de Radiologie 2001, 84:143-146.

9. Sadowski M, Tang CY, Aguinaldo JG, Carp R, Meeker HC, Wisniewski T: In vivo micro magnetic resonance imaging signal changes in scrapie infected mice. Neurosci Lett 2003, 345:1-4

10. Chung YL, Williams A, Beech JS, Williams SC, Bell JD, Cox IJ, Hope J: MR assessment of the blood-brain barrier in a hamster model of scrapie. Neurodegeneration 1995, 4:203-207.

11. Chung YL, Williams A, Ritchie D, Williams SC, Changani KK, Hope J, Bell JD: Conflicting MRI signals from gliosis and neuronal vacuolation in prion diseases. Neuroreport 1999, 10:3471-3477.

12. Haik $S$, Dormont D, Faucheux BA, Marsault C, Hauw JJ: Prion protein deposits match magnetic resonance imaging signal abnormalities in Creutzfeldt-Jakob disease. 2002, 797-799.

13. Bender S, Alverson J, Herrmann LM, O'Rourke KI: Histamine as an aid to biopsy of third eyelid lymphoid tissue in sheep. Vet Rec 2004 154:662-663.

14. O'Rourke Kl, Baszler TV, Besser TE, Miller JM, Cutlip RC, Wells GA, Ryder SJ, Parish SM, Hamir AN, Cockett NE, et al: Preclinical diagnosis of scrapie by immunohistochemistry of third eyelid lymphoid tissue. Journal of Clinical Microbiology 2000, 38:3254-3259.

15. Hosmer DW, Lemeshow S: Applied Logistic Regression. New York: John Wiley \& Sons, Inci, Second 2000

16. Miller JM, Jenny AL, Taylor WD, Race RE, Ernst DR, Katz JB, Rubenstein R Detection of prion protein in formalin-fixed brain by hydrated autoclaving immunohistochemistry for the diagnosis of scrapie in sheep. J Vet Diagn Invest 1994, 6:366-368.
17. Forstl H, Zerfass R, Geiger-Kabisch C, Sattel H, Besthorn C, Hentschel F: Brain atrophy in normal ageing and Alzheimer's disease. Volumetric discrimination and clinical correlations. Br J Psychiatry 1995, 167:739-746.

18. Liu C, Edwards S, Gong Q, Roberts N, Blumhardt LD: Three dimensional MRI estimates of brain and spinal cord atrophy in multiple sclerosis. 1999

19. Losseff NA, Wang L, Lai HM, Yoo DS, Gawne-Cain ML, McDonald Wl, Miller DH, Thompson AJ: Progressive cerebral atrophy in multiple sclerosis. A serial MRI study. Brain 1996, 119(Pt 6):2009-2019.

20. Yoshihara M, Tsunoda A, Sato K, Kanayama S, Calderon A: Differential diagnosis of NPH and brain atrophy assessed by measurement of intracranial and ventricular CSF volume with 3 D FASE MRI. Acta Neurochir Suppl 1998, 71:371-374.

21. Thompson AJ: Identification of brain atrophy with MRI in MS. Mult Scler 1998, 4:257-259.

22. van Keulen $L$, Vromans ME, van Zijderveld FG: Early and late pathogenesis of natural scrapie infection in sheep. APMIS 2002, 110:23-32.

23. van Keulen $\sqcup$, Schreuder BE, Vromans ME, Langeveld JP, Smits MA: Pathogenesis of natural scrapie in sheep. Archives of Virology Supplementum 2000, 57-71.

24. Andreoletti O, Berthon P, Marc D, Sarradin P, Grosclaude J, van Keulen L, Schelcher F, Elsen JM, Lantier F: Early accumulation of $\operatorname{PrP}(\mathrm{Sc})$ in gutassociated lymphoid and nervous tissues of susceptible sheep from a Romanov flock with natural scrapie. Journal of General Virology 2000, 81:3115-3126.

25. Ersdal C, Ulvund MJ, Benestad SL, Tranulis MA: Accumulation of pathogenic prion protein (PrPSc) in nervous and lymphoid tissues of sheep with subclinical scrapie. Veterinary Pathology 2003, 40:164-174.

26. Wood JL, McGill IS, Done SH, Bradley R: Neuropathology of scrapie: a study of the distribution patterns of brain lesions in 222 cases of natural scrapie in sheep, 1982-1991. Vet Rec 1997, 140:167-174.

27. Wood JL, McGill IS, Done SH, Bradley R: Neuropathology of scrapie: a study of the distribution patterns of brain lesions in 222 cases of natural scrapie in sheep, 1982-1991. Veterinary Record 1997, 140:167-174.

28. Hamir AN, Miller JM, Schmerr MJ, Stack MJ, Chaplin MJ, Cutlip RC Diagnosis of preclinical and subclinical scrapie in a naturally infected sheep flock utilizing currently available postmortem diagnostic techniques. Journal of Veterinary Diagnostic Investigation 2001, 13:152-154.

29. Ryder SJ, Spencer YI, Bellerby PJ, March SA: Immunohistochemical detection of PrP in the medulla oblongata of sheep: the spectrum of staining in normal and scrapie-affected sheep. Veterinary Record 2001, 148:7-13.

30. Kim H, O'Rourke Kl, Walter M, Purchase HG, Enck J, Shin TK Immunohistochemical detection of scrapie prion proteins in clinically normal sheep in Pennsylvania. Journal of Veterinary Diagnostic Investigation 2001, 13:89-91

doi:10.1186/1479-5876-8-125

Cite this article as: McKnight et al:: Generalized cerebral atrophy seen on MRI in a naturally exposed animal model for creutzfeldt-jakob disease. Journal of Translational Medicine 2010 8:125.

\section{Submit your next manuscript to BioMed Central and take full advantage of:}

- Convenient online submission

- Thorough peer review

- No space constraints or color figure charges

- Immediate publication on acceptance

- Inclusion in PubMed, CAS, Scopus and Google Scholar

- Research which is freely available for redistribution 